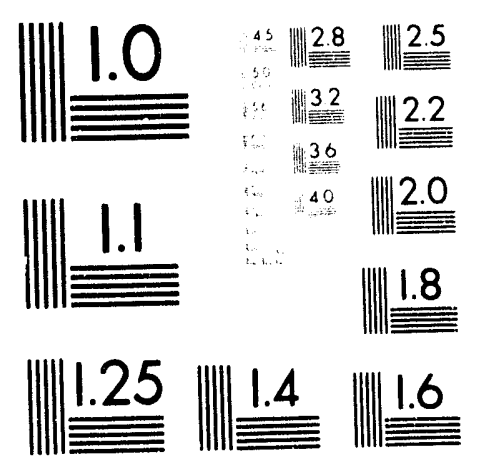



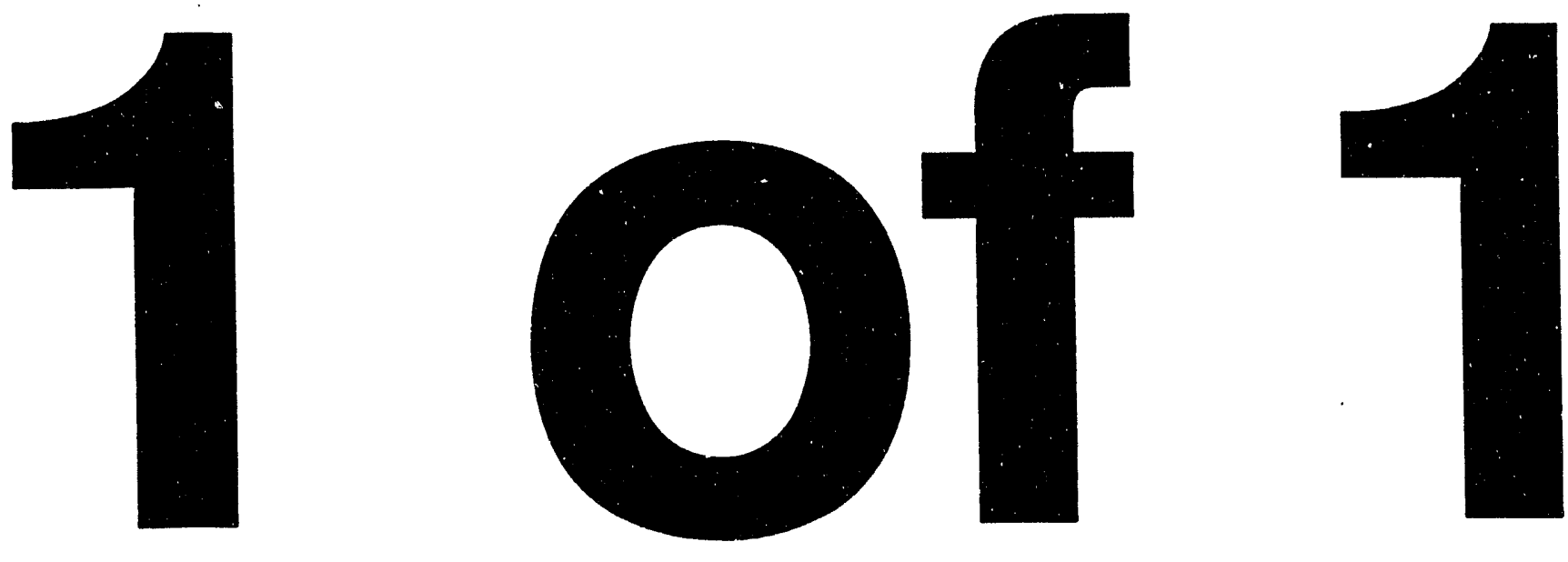


\title{
Mechanical Results of the CEBAF Cavity Series Production*
}

\author{
J. Mammosser and J.F. Benesch \\ Continuous Electron Beam Accelerator Facility \\ 12000 Jefferson Avenue, Newport News, VA 23606 USA
}

\section{Abstract}

Interface dimensions of the full production run of 360 CEBAF cavites manufactured by Siemens are summarized. Analysis indicates that length tolerances of $\pm 2 \mathrm{~mm}$ and fundamental power coupler location tolerances of $\pm 0.15 \mathrm{~mm}$ are achievable on future procurements. Vacuum leaks were a concern early in production but have now been overcome: $2 \mathrm{~K}$ helium leak rates integrated over 22 vacuum seals have been measured on 84 cavity pairs and a roughly normal distribution of the $\log 10$ (leak rate) is seen, centered about a rate of $10^{-10.2}$ torr-1/s.

\section{INTRODUCTION}

The Continuous Electron Beam Accelerator Facility (CEBAF) is under construction in Newport News, Virginia, USA The machine will produce a low emittance electron beam with a current of $200 \mu \mathrm{A}$ and energies up $105+\mathrm{GeV}$ for fundamental experimental studies in nuclear physics. [1] The accelerator consists of: a conventional source at $0.5 \mathrm{MeV}$, a single pass injection accelerator with nine pair of superconducting radio frequency (SRF) cavities providing final energy of $45 \mathrm{MeV}$, and a main ring in the shape of a racetrack with two linacs. The two linacs each consist of 80 pair of SRF cavities nominally providing $5 \mathrm{MV}$ each, for a total of $400 \mathrm{MeV} / \mathrm{inac}$ and $4 \mathrm{GeV}$ after five passes through each of the two linacs. There are thus 338 SRF cavities in the machine. A total of 360 cavities were purchased from Siemens.

Cavities are measured upon receipt and sorted into pairs according to window height $(C)$, overall length $(A)$ and window flange location $(B)$, with that order of precedence. About one third of the assemblies require custom interface components. About $10 \%$ require custom beam tubes to meet the cryostal length interface and another $\sim 20 \%$ require custom waveguide interfaces at (C).

\section{MECHANICAL TOLERANCES}

The outline of the CEBAF cavity, designed at Comell University (2), is shown in Figure 1.

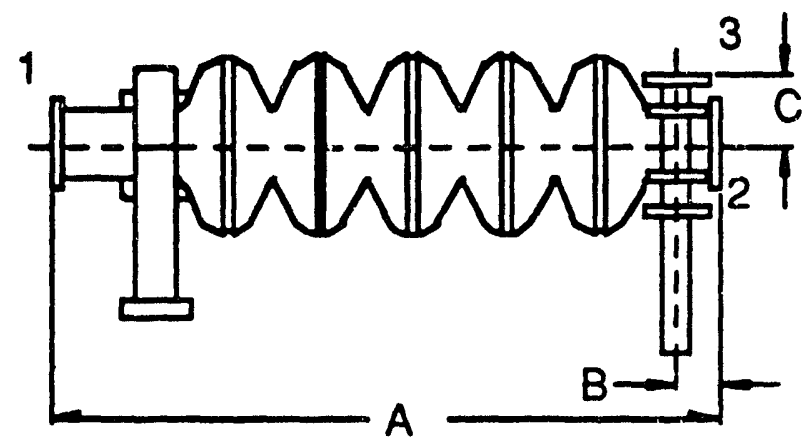

Figure 1. CEBAF cavity

Table 1 - Interface dimensions and tolerances (mm)

Overall length $(A)$

Coupler location (B)

Coupler height (C)

Perpendicularity of flanges $(1,2)$ to beam axis

Parallelism of coupler flange (3) to beam 0.2 axis

Specifications are in Table 1. Means and standard deviations achieved on the three lengths shown, as measured by CEBAF and Siemens, are given in Tables 2 and 3. Table 2 represents the furst 36 units (10\%) and Table 3 the remaining 324 units. The latter are more representative of full series production, and will be discussed in what follows.

Table 2 - Lengths (mm) - Cavities 1-36

Overall length $(A)$

CEBAF measurements $\quad 720.74 \quad 1.73$

Siemens

$720.44 \quad 1.57$

Window flange location (B)

CEBAF

Siemens

$46.33 \quad 0.14$

Window height (C)

CEBAF

Siemens

$46.35-0.05$

NA NA

$76.12 \quad 0.12$

Table 3 - Lengths (mm) - Cavities 37 and higher

Overall length $(A)$

$\begin{array}{lll}\text { CEBAF measurements } & 721.48 & 0.97\end{array}$

Siemens

$\begin{array}{ll}721.47 & 0.98\end{array}$

Window flange location (B)

CEBAF

$\begin{array}{ll}46.28 & 0.07\end{array}$

Siemens

46.32

0.05

Window height (C)

CEBAF

$76.28 \quad 0.06$

Siemens

76.25

0.07

The agreement between the measurements made of overall length is excellent. Only six cavities (2\%) fall outside $\pm 2 \sigma$. Since this performance was achieved even though the span allowed was much broader, a length tolerance of $\pm 2 \mathrm{~mm}$ is reasonable for future acquisitions.

Agreement between CEBAF and Siemens measurements on dimensions $B$ and $C$ is not as good. It is believed that this is due to the difference in the way the cavities were supported on the table of each organization's coordinate measuring machine. CEBAF supported the cavity at the first and fifth cells while Siemens supported three of the five cells. Cavities are held by one beam wbe and the coupler flange in the

- supported by U.S. DOE contract DE-ACO5-84ER40150. 
cryostat, so the CEBAF measurement is more representative gives the angles at points 1,2 and 3 on Figure 1. Future acquisitions should include explicit descriptions of the measurement setup to be used, including supports.

\section{Table 4 - Interface Angles}

mean

$\boldsymbol{\sigma}$

Beam flange angle at FPC (2)

$89.93^{\circ} 0.04^{\circ}$

$89.92^{\circ} \quad 0.04^{\circ}$ of use. The cavities sagged. This is shown in Table 4, which

Beam tlange angle at HOM (1)

Coupler flange angle to beam (3)

least 24 hours and is not pumped during this period, by monitoring the partial pressure of helium in the cavity vacuum as the cavity pair is warmed from $4.2 \mathrm{~K}$ w $20 \mathrm{~K}$ we can determine the total quantity of helium which leaked into the system while it was immersed. The distribution of the test results is shown in Figure 2.

The data is consistent (Shapiro-Wilk W tesh, see reference 4) with a nomal distribution with mean equal to -10.24 $\left(6 \times 10^{-11}\right.$ torr-l/s) and standard deviation just under an order of magnitude.

\section{log leak rate}

Looser tolerances for dimensions B and C would not require custom machining of interface components if one maintains an inventory of 40 to 50 cavities and sorts. Tolerances of $\pm 0.15 \mathrm{~mm}$ and $\pm 0.12 \mathrm{~mm}$ respectively are deemed achievable for future acquisitions.

The thinness ( $9 \mathrm{~mm}$ ) of the flanges of the design did not allow for adequate machining after welding to obtain better length tolerances, better perpendicularity of flanges 1 and 2 and better parallelism of flange 3 to the beam axis. The thinness of the flanges also has been implicated in problems with the integrity of the indium vacuum seals used in the assembly of the cavity pairs (see below). An increase in flange thickness to $15 \mathrm{~mm}$ before final machining would stiffen the flange by a factor of three and allow $0.7 \mathrm{~mm}$ of material for final machining to form and position. With thicker flanges, the form tolerances in table 1 (parallelism and perpendicularity) could be tightened from $0.2 \mathrm{~mm} 100.1 \mathrm{~mm}$, perhaps eliminating the need for custom interface components altogether. The interface parts could also include bellows.

\section{INDIUM VACUUM JOINTS}

During early production there were a number of leaks in the indium wire seals used as vacuum joints between the cavities and auxiliary components. The vacuum leaks were traced to two causes: (a) inappropriate final surface finishing and (b) deformation of flanges due to flange thickness, bolt hole pattem and bolt torquing procedure. Cause (a) was eliminated by electropolishing stainless steel flanges and lapping of the niobium flanges before chemical etching with a buffered solution of nitric, hydrofluoric and phosphoric acid. Cause (b) was eliminated by modifying the RF window frame $t$ increase stiffness, decreasing the indium wire size from 1.5 $\mathrm{mm}$ to $1.0 \mathrm{~mm}$ to reduce bolt torque, moving the indium wire seal towards the bolt holes and adopting new assembly and torquing procedures.

There are eighteen indium joints and four Conflato-style joints in each cavity pair assembly. After two cavities are assembled into a pair with hermetic RF windows, HOM loads and interfaces to the helium vessel, the pair is evacuated and maintained under vacuum thereafter. (3) The pair is mounted on a vertical test stand and placed in a dewar. RF testing is then performed at $2.0 \mathrm{~K}$. If a cavity pair does not meet the final vacuum leak specification at $2 \mathrm{~K}\left(<2 \times 10^{-8} \mathrm{std} \mathrm{cc} / \mathrm{s}\right)$ or if there is concern about RF coupler window appearance after the test, then cavity components may be replaced or the cavity pair may be reprocessed. In either case, the pair is retested and only the final results used. The leak rate is tested by an integration method: since the cavity is immersed in LHe for at

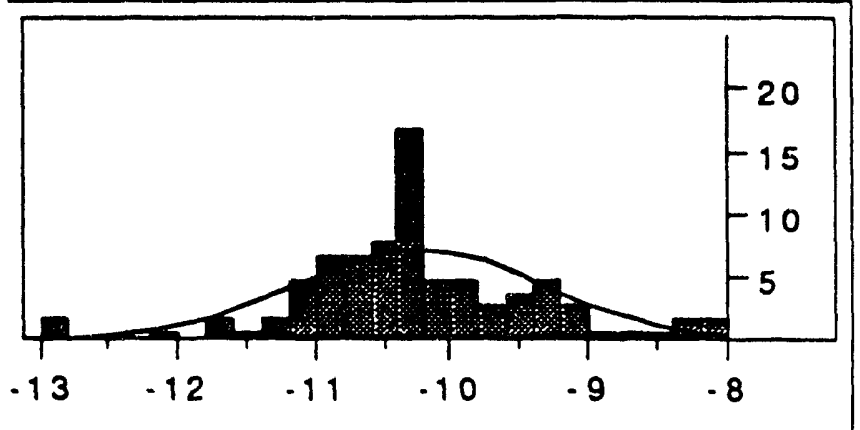

Figure 2. Common log of cold leak rate vs. number of cavities.

We are still having an occasional problem with cold RF window hermeticity. In addition, we have had a few leaks in indium joints due to oxides. Components can be removed and replaced without significant degradation of cavity performance by retuming the pair to the clean room, holding it in the vertical orientation, bleeding it up slowly to about 105 $\mathrm{kPa}$ with nitrogen through a point of use particle filter, removing external particles with solvent wiping and then carefully replacing the failed component or seal. Nitrogen flow through the pair and out the opening created by the part swap is maintained while the change is made to minimize particle influx. This procedure has been quite successful as is shown in figure 3, a case where the cold RF window was found to leak after vertical test. The two curves show the results of two distinct vertical tests, before and after the window change.

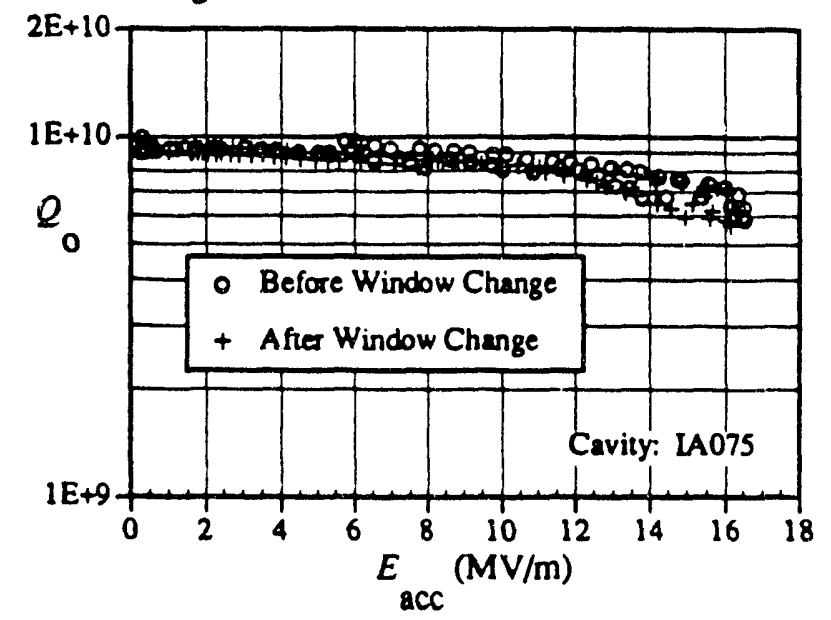

Figure 3. Durability of cavity performance with exchange of cold window 


\section{ACKNOWLEDGMENTS}

Cavity measurements at CEBAF were made by $K$. Yopp, H. Ward, A. Forrest and I. Daniels. Figure I was provided by K. Yopp and figure 3 by C. Reece.

\section{REFERENCES}

[1] H. Grunder, Proceeding of the 1988 Linear Accele rator Conference, and CEBAF report 89-001.

[2] P. Kneisel et al., Proceedings of 1991 Particle Accelerator Conference, $91 \mathrm{CH} 3038-7$, p. 2384.

[3] P. Kneisel, Proceedings of the 1987 Particle Accelerator Conference, CH2387-9/87, p. 1893.

[4] S.S Shapiro and M.B. Wilk, Biometrika 52, 591 (1965). 

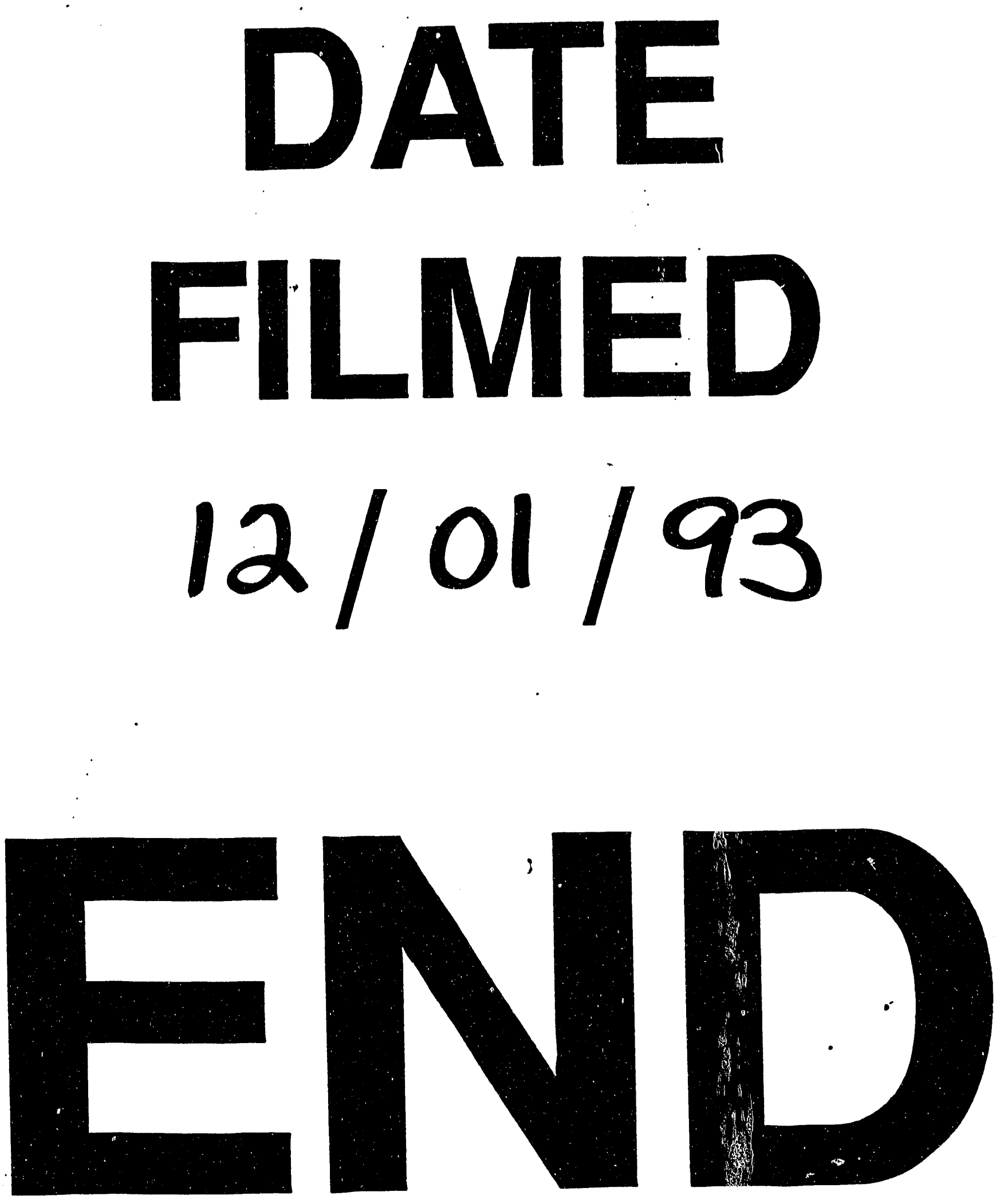
